\title{
CTX-M-14 and CTX-M-15 enzymes are the dominant type of extended-spectrum $\beta$-lactamase in clinical isolates of Escherichia coli from Korea
}

Correspondence

Seok Hoon Jeong

kscpjsh@yuhs.ac

Received 30 June 2008

Accepted 27 October 2008

\author{
Wonkeun Song, ${ }^{1}$ Hyukmin Lee, ${ }^{2}$ Kyungwon Lee, ${ }^{3}$ Seok Hoon Jeong, ${ }^{3}$ \\ II Kwon Bae, ${ }^{4}$ Jae-Seok Kim ${ }^{5}$ and Hyo-Sun Kwak ${ }^{6}$
}

\footnotetext{
${ }^{1}$ Department of Laboratory Medicine, Hallym University College of Medicine, 150-950, 948-1 Daerim 1-Dong, Youngdeungpo-Gu, Seoul, Republic of Korea

${ }^{2}$ Department of Laboratory Medicine, Kwandong University College of Medicine, 697-24 WhajungDong, Dukyang-Gu, Goyang, Republic of Korea

${ }^{3}$ Department of Laboratory Medicine and Research Institute of Bacterial Resistance, Yonsei University College of Medicine, 120-752, 134 Shinchon-Dong, Seodaemu-Gu, Seoul, Republic of Korea

${ }^{4}$ Research Institute for Antimicrobial Resistance, Kosin University College of Medicine, 602-030, 34 Amnam-Dong, Suh-Gu, Busan, Republic of Korea

${ }^{5}$ Department of Laboratory Medicine, Hallym University College of Medicine, 134-701, 150 Sungnae-Gil, Kangdong-Gu, Seoul, Republic of Korea

${ }^{6}$ Center for Food Safety Evaluation, Korea Food and Drug Administration, 122-704, 231 JinheungRo, Eunpyung-Gu, Seoul, Republic of Korea
}

This study was performed to assess the prevalence and genotypes of plasmid-borne extendedspectrum $\beta$-lactamases (ESBLs) and AmpC $\beta$-lactamases in Escherichia coli in Korea. A total of 576 isolates of $E$. coli was collected from 12 Korean hospitals during May and July 2007. A phenotypic confirmatory test detected ESBLs in $82(14.2 \%)$ of the $576 \mathrm{E}$. coli isolates. The most common types of ESBLs identified were CTX-M-14 $(n=32)$ and CTX-M-15 $(n=27)$. The prevalence and diversity of the CTX-M mutants, including CTX-M-15, CTX-M-27 and CTX-M-57, with significant hydrolytic activity against ceftazidime were increased. PCR experiments detected genes encoding plasmid-borne AmpC $\beta$-lactamases in 15/56 cefoxitin-intermediate or cefoxitinresistant isolates, and the most common type of $\operatorname{AmpC} \beta$-lactamase identified was DHA-1 $(n=10)$. These data suggest that the incidence of ESBLs in E. coli has increased as a result of the dissemination of CTX-M enzymes in Korea. In addition, CTX-M-22, CTX-M-27 and CTX-M-57 have appeared in Korea.

\section{INTRODUCTION}

The predominant mechanism for acquired resistance to $\beta$ lactams in Escherichia coli is the synthesis of plasmid-borne extended-spectrum $\beta$-lactamases (ESBLs) and AmpC $\beta$ lactamases. The clavulanic acid (CA)-inhibitory ESBLs are within molecular class A of the Ambler classification scheme and confer resistance to oxyimino-cephalosporins on their bacterial hosts (Paterson \& Bonomo, 2005). CTX$M$ enzymes are spreading rapidly and are now the dominant type of ESBL in E. coli in many parts of the world (Rossolini et al., 2008), although classical TEM and SHV ESBLs are still dominant in the USA (Bush, 2008). In

Abbreviations: BA, boronic acid; CA, clavulanic acid; CAZ, ceftazidime; CTX, cefotaxime; ESBL, extended-spectrum $\beta$-lactamase; FOX, cefoxitin.
Korea, members of the CTX-M-1 and CTX-M-9 clusters have repeatedly been reported in Enterobacteriaceae since the first finding of CTX-M-14 in clinical isolates of Shigella sonnei, E. coli and Klebsiella pneumoniae in 2001 (Pai et al., 2001). A nationwide survey in 2003 reported 23/246 clinical isolates $(9.3 \%)$ of E. coli with an ESBL phenotype, and only 8 of these had CTX-M enzymes (Ryoo et al., 2005).

E. coli is intrinsically susceptible to $7-\alpha$-methoxy-cephalosporins [e.g. cefoxitin (FOX) and cefotetan] because of the low-level expression of the non-inducible species-specific ampC gene (Philippon et al., 2002). In 1989, Bauernfeind et al. (1989) described a FOX-resistant K. pneumoniae isolate, which produced a plasmid-borne AmpC $\beta$ lactamase, named CMY-1, from Korea. Subsequently, various types of plasmid-borne AmpC enzymes have been 
found worldwide (Philippon et al., 2002). In Korea, FOXresistant $E$. coli have increasingly been noted, and a study performed in 2003 showed that a high portion $(53.4 \%, 62 /$ 116) of FOX resistance in E. coli was due to plasmid-borne AmpC $\beta$-lactamase production (Lee et al., 2006). The aim of the present study was to determine the prevalence and shift of plasmid-borne AmpC $\beta$-lactamases and ESBLs, with a special focus on the CTX-M enzymes, in E. coli in Korea.

\section{METHODS}

Bacterial strains. Consecutive non-duplicate isolates of E. coli were collected during May and July 2007 from 12 hospitals in nine cities in Korea. The isolates were identified using the API-20 E system (bioMérieux). The azide-resistant E. coli J53 was used as a recipient strain for conjugative transfer. E. coli ATCC 25922 and K. pneumoniae ATCC 700603 were used as MIC reference strains.

Antimicrobial susceptibility testing. Antibiotic-containing discs (BBL) were used for routine antibiograms by disc diffusion assay (CLSI, 2006a). The phenotypic confirmatory test for ESBL and/or AmpC $\beta$ lactamase production using boronic acid (BA) as an AmpC $\beta$-lactamase inhibitor was performed as described previously (Song et al., 2007). MICs were determined by the agar dilution method using Mueller-
Hinton agar (MHA; Difco Laboratories) with an inoculum of $10^{4}$ c.f.u. (CLSI, 2006b). The MICs of $\beta$-lactams were determined alone or in combination with a fixed concentration $\left(4 \mu \mathrm{g} \mathrm{ml}^{-1}\right)$ of CA.

Mating-out assays. Conjugation experiments were carried out between donors and the azide-resistant recipient strain E. coli J53 on MHA plates. Transconjugants were selected on MHA plates supplemented with $2 \mu \mathrm{g}$ ceftazidime $(\mathrm{CAZ}) \mathrm{ml}^{-1}$ or $2 \mu \mathrm{g}$ cefotaxime (CTX) $\mathrm{ml}^{-1}$ and $100 \mu \mathrm{g}$ sodium azide $\mathrm{ml}^{-1}$.

Characterization of genes encoding $\boldsymbol{\beta}$-lactamases. Detection of genes encoding plasmid-borne ESBLs and AmpC $\beta$-lactamases was performed by PCR amplification with the primers listed in Table 1, as described previously (Ryoo et al., 2005; Song et al., 2006). Templates for PCR amplification in the clinical isolates were plasmid preparations from the clinical isolates. The PCR products were subjected to direct sequencing. Both strands of all PCR products were sequenced twice using an automatic sequencer (model 3730xl; Applied Biosystems).

\section{RESULTS}

\section{E. coli isolates harbouring ESBLs}

In the study period, clinical E. coli isolates $(n=576)$ were obtained from outpatients $(46.2 \%)$, inpatients of general

Table 1. Primers used in this study

\begin{tabular}{|c|c|c|}
\hline PCR target & Primer name & Primer sequence $\left(5^{\prime} \rightarrow 3^{\prime}\right)$ \\
\hline \multirow[t]{4}{*}{$b l a_{\text {СТХ-М }}($ CTX-M-1 cluster $)$} & CTX-M-1F & CCGTCACGCTGTTGTTAGG \\
\hline & CTX-M-1R & GACGATTTTAGCCGCCGAC \\
\hline & $\mathrm{BM}-1 \mathrm{~F}$ & ACTATGGCACCACCAACGAT \\
\hline & FM-1R & TTCGGTTCGCTTTCACTTTT \\
\hline \multirow[t]{2}{*}{$b l a_{\mathrm{CTX}-\mathrm{M}}(\mathrm{CTX}-\mathrm{M}-2$ cluster $)$} & CTX-M-2F & CGGTGCTTAAACAGAGCGAG \\
\hline & CTX-M-2R & CCATGAATAAGCAGCTGATTGCCC \\
\hline \multirow[t]{2}{*}{$b l a_{\mathrm{CTX}-\mathrm{M}}(\mathrm{CTX}-\mathrm{M}-8$ cluster $)$} & CTX-M-8F & ACGCTCAACACCGCGATC \\
\hline & CTX-M-8R & CGTGGGTTCTCGGGGATAA \\
\hline \multirow[t]{2}{*}{$b l a_{\text {СTX-M }}($ CTX-M-9 cluster $)$} & CTX-M-9F & GATTGACCGTATTGGGAGTTT \\
\hline & CTX-M-9R & CGGCTGGGTAAAATAGGTCA \\
\hline \multirow[t]{2}{*}{$b l a_{\mathrm{TEM}}$} & TEM-F & ATGAGTATTCAACATTTCCGT \\
\hline & TEM-R & TTACCAATGCTTAATCAGTGA \\
\hline \multirow[t]{2}{*}{$b l a_{\mathrm{SHV}}$} & SHV-F & CCGGGTTATTCTTATTTGTCGCT \\
\hline & SHV-R & TAGCGTTGCCAGTGCTCG \\
\hline \multirow[t]{2}{*}{$b l a_{\mathrm{PER}-1}$} & PER-1F & GTTAATTTGGGCTTAGGGCAG \\
\hline & PER-1R & CAGCGCAATCCCCACTGT \\
\hline \multirow[t]{2}{*}{$b l a_{\mathrm{VEB}}$} & VEB-F & ACCAGATAGGAGTACAGACATATGA \\
\hline & VEB-R & TTCATCACCGCGATAAAGCAC \\
\hline \multirow[t]{2}{*}{$b l a_{\mathrm{GES} / \mathrm{IBC}}$} & GES/IBC-F & GTTAGACGGGCGTACAAAGATAAT \\
\hline & GES/IBC-R & TGTCCGTGCTCAGGATGAGT \\
\hline \multirow[t]{2}{*}{$b l a_{\mathrm{TLA}}$} & TLA-F & CGCGAAAATTCTGAAATGAC \\
\hline & TLA-R & AGGAAATTGTACCGAGACCCT \\
\hline \multirow[t]{2}{*}{$b l a_{\mathrm{DHA}-1}$} & DHA-F & GGGGAGATAACGTCTGACCA \\
\hline & DHA-R & TAGCCAGATCCAGCAATGTG \\
\hline \multirow[t]{2}{*}{$b l a_{\mathrm{CMY}-1}$} & CMY-1F & TCACATCGGCTTCACAGAGC \\
\hline & CMY-1R & CCATGGTGATGCTGTCAAAGA \\
\hline \multirow[t]{2}{*}{$b l a_{\mathrm{CMY}-2}$} & CMY-2F & CAACACGGTGCAAATCAAAC \\
\hline & CMY-2R & CATGGGATTTTCCTTGCTGT \\
\hline \multirow{2}{*}{$b l a_{\mathrm{ACT}-1}$} & ACT-1F & CGTCATGGTCTCGTCCGTTAG \\
\hline & ACT-1R & CCTTGACCTCATCCGGTACCT \\
\hline
\end{tabular}


wards $(46.9 \%)$ and inpatients of intensive care units $(6.9 \%)$. These isolates were obtained from urine $(70.3 \%)$, blood $(8.3 \%)$, wound $(7.4 \%)$, sputum $(6.2 \%)$ and other samples $(7.8 \%)$. The phenotypic confirmatory test with BA detected ESBLs in $82(14.2 \%)$ E. coli isolates. Clinical isolates with an ESBL phenotype were found in all 12 hospitals (Table 2). Transfer of CAZ or CTX resistance determinants to the azide-resistant $E$. coli J53 recipient by conjugation was successful in 56/82 isolates with an ESBL phenotype.

PCR experiments detected genes encoding members of the CTX-M-1 and CTX-M-9 clusters in 42 and 35 isolates, respectively. The most common types of class A ESBLs identified were CTX-M-14 $(n=32)$ and CTX-M-15 $(n=27)$. Genes encoding CTX-M-3 $(n=10)$, CTX-M-9 $(n=2)$, CTX-M-12 $(n=1)$, CTX-M-22 $(n=2)$, CTX-M-27 $(n=1)$ and CTX-M-57 $(n=2)$ were also detected. The bla gene of SHV-12 was detected in two isolates. Genes encoding TEM-type $\beta$-lactamases were detected in 62/82 isolates, but all were TEM-1. Non-TEM and non-SHV ESBLs, including PER, VEB, GES and TLA enzymes and members of the CTX-M-2 and CTX-M-8 clusters, were not detected in this survey. No ESBL was detected in $4 / 82$ isolates with an ESBL phenotype. These isolates may have another ESBL-encoding gene not detected with our primers or may have had false-positive results for ESBL activity.

\section{E. coli isolates harbouring AmpC $\beta$-lactamases}

Among 56 FOX-intermediate or FOX-resistant isolates, 26 exhibited positive results in the phenotypic confirmatory test, i.e. $a \geqslant 5 \mathrm{~mm}$ increase in the zone diameter of either the FOX or the cefotetan disc in the presence of BA. PCR experiments detected genes encoding plasmid-borne AmpC $\beta$-lactamases in 15 isolates. The most common type of AmpC $\beta$-lactamase identified was DHA-1 $(n=10)$, and genes encoding CMY-2 $(n=3)$, CMY-10 $(n=1)$ and CMY-11 $(n=1)$ were also detected. Nine of the fifteen isolates simultaneously harboured ESBLs.

\section{Phenotypic characteristics}

All of the isolates producing CTX-M-3 ( $n=10)$, CTX-M-9 $(n=1)$, CTX-M-14 $(n=30)$ or CTX-M-22 $(n=1)$ had more than eightfold higher MICs for CTX than for CAZ (Table 3). The isolates producing CTX-M-15, CTX-M-27 or CTX-M-57 exhibited a high level of resistance to CAZ. Fourteen isolates producing plasmid-borne AmpC $\beta$ lactamases were highly resistant to FOX (MICs $\geqslant 128 \mathrm{mg}$ $1^{-1}$ ), except for two producing DHA-1. All of the isolates producing ESBLs exhibited a high level of resistance to ciprofloxacin (MICs $\geqslant 64 \mathrm{mg} \mathrm{l}^{-1}$ ), except for three (data not shown). A total of 15 out of 82 isolates $(18.3 \%$ ) with an ESBL phenotype were resistant to amikacin (MICs $\geqslant 32 \mathrm{mg} \mathrm{l}^{-1}$ ).

\section{DISCUSSION}

Compared with a survey in 1997 (Pai et al., 1999), the prevalence of ESBL-producing E. coli in Korea has increased threefold from 4.8 to $14.2 \%$. We have shown previously that only $3.3 \%(8 / 246)$ of clinical E. coli isolates produced CTX-M ESBLs in 2003 (Ryoo et al., 2005), but the prevalence of these enzymes increased to $13.4 \%$ (77/576) in the present study. These results indicate that the significant increase in ESBL incidence in E. coli may be due to dissemination of CTX-M enzymes. The incidence of CTX-M-14 and CTX-M-15 increased from $0.4 \%(1 / 246)$ and $1.6 \%(4 / 246)$ to $5.6 \%(32 / 576)$ and $4.7 \%(27 / 576)$, respectively, during this period (Ryoo et al., 2005).

Dissemination of E. coli harbouring members of the CTXM-1 and CTX-M-9 clusters has repeatedly been reported in Asian countries (Hawkey, 2008). CTX-M-14 was the dominant type of ESBL in E. coli from China (223/325) and Taiwan (88/128) (Yan et al., 2006; Yu et al., 2007), and CTX-M-3 was the second most frequent type in these countries (53/325 and 33/128, respectively). Interestingly, CTX-M-15 was rarely detected in these countries $(2 / 325$ and $2 / 128$, respectively), whilst the enzyme was the second most dominant type of ESBL in Korea. Three CTX-M enzymes, including CTX-M-22, CTX-M-27 and CTX-M57 , were detected for what is believed to be the first time in Korea in the present study.

It is worth noting that the prevalence and diversity of the CTX-M mutants, including CTX-M-15, CTX-M-27 and CTX-M-57, with significant hydrolytic activity against CAZ have increased (Poirel et al., 2002; Bonnet et al., 2003; Hopkins et al., 2008). Past reports have shown that the most common ESBL in E. coli isolates from Korea was TEM-52 (Pai et al., 1999), but this ESBL was not detected in our study. Furthermore, SHV-12, which was the most common ESBL in K. pneumoniae in 2003 (Ryoo et al., 2005), was detected in only two isolates in this study. Thus, it appears that CTX-M enzymes with an expanded activity towards CAZ in E. coli may be replacing TEM-52 and SHV12 , which confers a high level of resistance against CAZ on their bacterial hosts.

A survey in 2003 showed that the most common plasmidborne AmpC $\beta$-lactamase in E. coli isolates from Korea was CMY-2 (Lee et al., 2006), but it was detected in only three isolates in this study. The present study showed that the most common AmpC $\beta$-lactamase in E. coli isolates was DHA-1, an inducible enzyme, and the proportion of AmpC $\beta$-lactamase-producing isolates among the ESBL-producing and -non-producing isolates was significantly different: $11.0 \%(9 / 82)$ versus $1.2 \%(6 / 494)$, respectively.

The present data suggest that the incidence of isolation of ESBLs in E. coli has increased as a result of the dissemination of CTX-M enzymes in Korea. The most common ESBL and AmpC $\beta$-lactamase in E. coli were CTX-M-14 and DHA-1, respectively. In addition, CTX-M22, CTX-M-27 and CTX-M-57 have appeared in Korea. 
Table 2. Distribution of ESBL and AmpC $\beta$-lactamases in $E$. coli isolates from Korea

\begin{tabular}{|c|c|c|c|c|c|c|c|c|c|c|c|c|c|}
\hline \multirow[t]{2}{*}{ Type of $\beta$-lactamase } & \multicolumn{12}{|c|}{ No. of isolates collected from each hospital } & \multirow{2}{*}{$\begin{array}{l}\text { Total no. of } \\
\text { isolates } \\
(n=576)\end{array}$} \\
\hline & $\begin{array}{c}\text { SL1 } \\
(n=40)\end{array}$ & $\begin{array}{c}\text { SL2 } \\
(n=49)\end{array}$ & $\begin{array}{c}\text { SL3 } \\
(n=50)\end{array}$ & $\begin{array}{c}\text { SL4 } \\
(n=50)\end{array}$ & $\begin{array}{c}\text { GY } \\
(n=50)\end{array}$ & $\begin{array}{c}\mathrm{SN} \\
(n=50)\end{array}$ & $\begin{array}{c}\text { SW } \\
(n=50)\end{array}$ & $\begin{array}{c}\text { WJ } \\
(n=50)\end{array}$ & $\begin{array}{c}\text { GM } \\
(n=49)\end{array}$ & $\begin{array}{c}\text { BS } \\
(n=50)\end{array}$ & $\begin{array}{c}\text { GJ } \\
(n=50)\end{array}$ & $\begin{array}{c}\mathrm{JJ} \\
(n=38)\end{array}$ & \\
\hline CTX-M-1 cluster & 5 & 3 & 2 & 3 & 1 & 3 & 3 & 5 & 4 & 5 & 5 & 3 & 42 \\
\hline CTX-M-3 & & & 1 & 1 & & 1 & & 3 & 2 & 1 & 1 & & 10 \\
\hline CTX-M-12+DHA-1 & & & & & & & & & & & 1 & & 1 \\
\hline CTX-M-15 & 5 & 2 & 1 & & 1 & 2 & 2 & 2 & 2 & 2 & 2 & 3 & 24 \\
\hline CTX-M-15 + DHA-1 & & & & 1 & & & & & & & & & 1 \\
\hline CTX-M-15 + CMY-2 & & & & & & & & & & & 1 & & 1 \\
\hline CTX-M-15 + CMY-10 & & & & & & & 1 & & & & & & 1 \\
\hline CTX-M-22 & & & & & & & & & & 1 & & & 1 \\
\hline CTX-M-22 + DHA-1 & & & & & & & & & & 1 & & & 1 \\
\hline CTX-M-57 & & 1 & & & & & & & & & & & 1 \\
\hline CTX-M-57 + DHA-1 & & & & 1 & & & & & & & & & 1 \\
\hline CTX-M-9 cluster & 2 & 4 & 4 & 2 & 1 & 0 & 6 & 8 & 2 & 3 & 1 & 2 & 35 \\
\hline CTX-M-9 & & & & & & & & & & 1 & & & 1 \\
\hline CTX-M-9+ DHA-1 & & & & & & & & & & 1 & & & 1 \\
\hline CTX-M-14 & 2 & 2 & 4 & 1 & 1 & & 6 & 8 & 2 & 1 & 1 & 2 & 30 \\
\hline CTX-M-14 + SHV-12 & & 1 & & & & & & & & & & & 1 \\
\hline CTX-M-14 + CMY-2 & & & & 1 & & & & & & & & & 1 \\
\hline CTX-M-27 & & 1 & & & & & & & & & & & 1 \\
\hline SHV-12+DHA-1 & & & & & & & 1 & & & & & & 1 \\
\hline DHA-1 & & 3 & 1 & & & & & & & & & & 4 \\
\hline CMY-2 & & & 1 & & & & & & & & & & 1 \\
\hline CMY-11 & & & & & & & & & & & 1 & & 1 \\
\hline Unidentified $\dagger$ & & & & 1 & 1 & & & & & 2 & & & 4 \\
\hline Total & 7 & 10 & 8 & 6 & 3 & 3 & 10 & 13 & 6 & 10 & 7 & 5 & 88 \\
\hline
\end{tabular}

*The 12 hospitals were in Seoul (SL1-4), Goyang (GY), Sungnam (SN), Suwon (SW), Wonju (WJ), Gumi (GM), Busan (BS), Gwangju (GJ) and Jeju (JJ) in Korea.

$\dagger$ The BA disc test for ESBLs was positive, but no ESBL or ampC genes were detected. 
Table 3. MICs $\left(\mu \mathrm{gl}^{-1}\right)$ for $82 \mathrm{E}$. coli isolates with ESBL and/or AmpC enzymes

\begin{tabular}{|c|c|c|c|c|c|c|c|c|c|c|c|c|c|c|c|}
\hline \multirow{2}{*}{$\begin{array}{l}\text { Type of } \beta \text {-lactamase } \\
\text { (no. of isolates) }\end{array}$} & \multicolumn{3}{|c|}{ FOX } & \multicolumn{3}{|c|}{ CAZ } & \multicolumn{3}{|c|}{$\mathrm{CAZ}-\mathrm{CA}^{*}$} & \multicolumn{3}{|c|}{ CTX } & \multicolumn{3}{|c|}{ CTX-CA* } \\
\hline & Range & $\mathrm{MIC}_{50}$ & $\mathrm{MIC}_{90}$ & Range & $\mathrm{MIC}_{50}$ & $\mathrm{MIC}_{90}$ & Range & $\mathrm{MIC}_{50}$ & $\mathrm{MIC}_{90}$ & Range & $\mathrm{MIC}_{50}$ & $\mathrm{MIC}_{90}$ & Range & $\mathrm{MIC}_{50}$ & $\mathrm{MIC}_{90}$ \\
\hline CTX-M-3 (10) & $4-32$ & 8 & 32 & $2-32$ & 4 & 32 & $0.3-1$ & 1 & 1 & $16->256$ & 128 & $>256$ & $0.1-1$ & 0.3 & 1 \\
\hline CTX-M-12 + DHA-1 (1) & 256 & & & 256 & & & 128 & & & 64 & & & 32 & & \\
\hline CTX-M-15 (24) & $4-64$ & 16 & 32 & $16->256$ & 64 & $>256$ & $0.3-4$ & 1 & 4 & $128->256$ & $>256$ & $>256$ & $0.1-1$ & 0.5 & 1 \\
\hline CTX-M-15 + DHA-1 (1) & 128 & & & 64 & & & 2 & & & $>256$ & & & 1 & & \\
\hline CTX-M-15 + CMY-2 (1) & 256 & & & 64 & & & 64 & & & $>256$ & & & 32 & & \\
\hline CTX-M-15 + CMY-10 (1) & $>256$ & & & 64 & & & 16 & & & 256 & & & 128 & & \\
\hline CTX-M-22 (1) & 2 & & & 1 & & & 0.1 & & & 32 & & & 0.1 & & \\
\hline CTX-M-22 + DHA-1 (1) & 256 & & & 32 & & & 2 & & & 32 & & & 1 & & \\
\hline CTX-M-57 (1) & 64 & & & 32 & & & 1 & & & 256 & & & 0.3 & & \\
\hline CTX-M-57 + DHA-1 (1) & 256 & & & 64 & & & 8 & & & 256 & & & 2 & & \\
\hline CTX-M-9 (1) & 4 & & & 1 & & & 0.3 & & & 32 & & & 0.1 & & \\
\hline CTX-M-9 + DHA-1 (1) & 128 & & & 2 & & & 1 & & & $>256$ & & & 0.5 & & \\
\hline CTX-M-14 (30) & $2-128$ & 16 & 64 & $0.3-16$ & 2 & 8 & $0.1-2$ & 1 & 2 & $16->256$ & 128 & 256 & $0.1-1$ & 0.5 & 1 \\
\hline CTX-M-14 + SHV-12 (1) & 16 & & & 256 & & & 1 & & & 64 & & & 0.1 & & \\
\hline CTX-M-14 + CMY-2 (1) & 256 & & & 64 & & & 64 & & & 128 & & & 32 & & \\
\hline CTX-M-27 (1) & 16 & & & 256 & & & 1 & & & $>256$ & & & 0.3 & & \\
\hline SHV-12 + DHA-1 (1) & $>256$ & & & 64 & & & 8 & & & 16 & & & 4 & & \\
\hline DHA-1 (4) & $16-256$ & & & $0.1-32$ & & & $0.1-8$ & & & $0.1-32$ & & & $0.1-8$ & & \\
\hline CMY-2 (1) & 256 & & & 16 & & & 16 & & & 16 & & & 32 & & \\
\hline CMY-11 (1) & $>256$ & & & 16 & & & 16 & & & 128 & & & 128 & & \\
\hline
\end{tabular}

${ }^{\star} \mathrm{CA}$ at a fixed concentration of $4 \mu \mathrm{g} \mathrm{ml}^{-1}$. 


\section{ACKNOWLEDGEMENTS}

We thank J. Y. Ahn, J. H. Shin. Y. Uh, W. G. Lee, M. N. Kim, Y. J. Park, S. H. Lee and S. G. Hong for providing clinical isolates of E. coli. This work was supported by a research grant from the Korea Food and Drug Administration (07052Hangsaengjae174).

\section{REFERENCES}

Bauernfeind, A., Chong, Y. \& Schweighart, S. (1989). Extended broad-spectrum $\beta$-lactamase in Klebsiella pneumoniae including resistance to cephamycins. Infection 17, 316-321.

Bonnet, R., Recule, C., Baraduc, R., Chanal, C., Sirot, D., De Champs, C. \& Sirot, J. (2003). Effect of D240G substitution in a novel ESBL CTX-M27. J Antimicrob Chemother 52, 29-35.

Bush, K. (2008). Extended-spectrum $\beta$-lactamases in North America, 1987-2006. Clin Microbiol Infect 14 (Suppl. 1), 134-143.

CLSI (2006a). Methods for Dilution Antimicrobial Susceptibility Tests for Bacteria that Grow Aerobically, 7th edn, approved standard M7A7. Wayne, PA: Clinical and Laboratory Standards Institute.

CLSI (2006b). Performance Standards for Antimicrobial Disk Susceptibility Tests, 9th edn, approved standard M2-A9. Wayne, PA: Clinical and Laboratory Standards Institute.

Hawkey, P. M. (2008). Prevalence and clonality of extended-spectrum $\beta$-lactamases in Asia. Clin Microbiol Infect 14 (Suppl. 1), 159-165.

Hopkins, K. L., Threlfall, E. J., Karisik, E. \& Wardle, J. K. (2008). Identification of novel plasmid-mediated extended-spectrum $\beta$ lactamase CTX-M-57 in Salmonella enterica serovar Typhimurium. Int J Antimicrob Agents 31, 85-86.

Lee, K., Lee, M., Shin, J. H., Lee, M. H., Kang, S. H., Park, A. J., Yong, D. \& Chong, Y. (2006). Prevalence of plasmid-mediated AmpC $\beta$ lactamases in Escherichia coli and Klebsiella pneumoniae in Korea. Microb Drug Resist 12, 44-49.

Pai, H., Lyu, S., Lee, J. H., Kim, J., Kwon, Y., Kim, J. W. \& Choe, K. W. (1999). Survey of extended-spectrum $\beta$-lactamases in clinical isolates of Escherichia coli and Klebsiella pneumoniae: prevalence of TEM-52 in Korea. J Clin Microbiol 37, 1758-1763.
Pai, H., Choi, E. H., Lee, H. J., Hong, J. Y. \& Jacoby, G. A. (2001). Identification of CTX-M-14 extended-spectrum $\beta$-lactamase in clinical isolates of Shigella sonnei, Escherichia coli, and Klebsiella pneumoniae in Korea. J Clin Microbiol 39, 3747-3749.

Paterson, D. L. \& Bonomo, R. A. (2005). Extended-spectrum $\beta$ lactamases: a clinical update. Clin Microbiol Rev 18, 657-686.

Philippon, A., Arlet, G. \& Jacoby, G. A. (2002). Plasmid-determined AmpC-type $\beta$-lactamases. Antimicrob Agents Chemother 46, 1-11.

Poirel, L., Gniadkowski, M. \& Nordmann, P. (2002). Biochemical analysis of the ceftazidime-hydrolysing extended-spectrum $\beta$-lactamase CTX-M-15 and of its structurally related $\beta$-lactamase CTX-M-3. J Antimicrob Chemother 50, 1031-1034.

Rossolini, G. M., D’Andrea, M. M. \& Mugnaioli, C. (2008). The spread of CTX-M-type extended-spectrum $\beta$-lactamases. Clin Microbiol Infect 14 (Suppl. 1), 33-41.

Ryoo, N. H., Kim, E. C., Hong, S. G., Park, Y. J., Lee, K., Bae, I. K., Song, E. H. \& Jeong, S. H. (2005). Dissemination of SHV-12 and CTX-M-type extended-spectrum $\beta$-lactamases among clinical isolates of Escherichia coli and Klebsiella pneumoniae and emergence of GES-3 in Korea. J Antimicrob Chemother 56, 698-702.

Song, W., Kim, J. S., Kim, H. S., Yong, D., Jeong, S. H., Park, M. J. \& Lee, K. M. (2006). Increasing trend in the prevalence of plasmidmediated AmpC $\beta$-lactamases in Enterobacteriaceae lacking chromosomal ampC gene at a Korean university hospital from 2002 to 2004. Diagn Microbiol Infect Dis 55, 219-224.

Song, W., Bae, I. K., Lee, Y. N., Lee, C. H., Lee, S. H. \& Jeong, S. H. (2007). Detection of extended-spectrum $\beta$-lactamases by using boronic acid as an AmpC $\beta$-lactamase inhibitor in clinical isolates of Klebsiella spp. and Escherichia coli. J Clin Microbiol 45, 1180-1184.

Yan, J. J., Hsueh, P. R., Lu, J. J., Chang, F. Y., Shyr, J. M., Wan, J. H., Liu, Y. C., Chuang, Y. C., Yang, Y. C. \& other authors (2006). Extended-spectrum $\beta$-lactamases and plasmid-mediated AmpC enzymes among clinical isolates of Escherichia coli and Klebsiella pneumoniae from seven medical centers in Taiwan. Antimicrob Agents Chemother 50, 1861-1864.

Yu, Y., Ji, S., Chen, Y., Zhou, W., Wei, Z., Li, L. \& Ma, Y. (2007). Resistance of strains producing extended-spectrum $\beta$-lactamases and genotype distribution in China. J Infect 54, 53-57. 